\title{
Experimental test of the optimal rotation for the finite stiffness cantilever rod
}

\author{
Aleksandr I. Bokhonsky ${ }^{1,}$, Mikhail M. Maistrishin ${ }^{1}$, and Aleksandr I. Ryzhkov ${ }^{1}$ \\ ${ }^{1}$ Sevastopol State University, Sevastopol, Russian Federation
}

\begin{abstract}
One of the simplest types of skew-symmetric optimal controls for the motion of an object as an absolutely rigid and deformable body was theoretically researched and experimentally verified. An elastic system with one degree of freedom was observed as an example, and it was shown, that for an acceptable minimum possible time an absolute rest could be achieved at the end of the motion. The time of motion was found from the system of transcendental equations, which are moment relations in relative motion (relative displacement and relative velocity at the end of translational motion are equal to zero). Experimental test was completed for the optimal translational rotation of the elastic cantilever rod with a distributed mass and the absolute rest was achieved after optimal turning through a predetermined angle (in the shortest possible time) around the axis passing through the end of the rod. Results of the research can find application in: optimal control of the motion of finite stiffness objects in automated production, creation of manipulators of a minimal mass, in the case of mounting non-rigid large-sized structures in outer space, in a number of other areas of modern technologies.
\end{abstract}

\section{Introduction}

Papers [1-7] and others are devoted to the theory of oscillations of elastic systems with a finite and infinite number degrees of freedom. The methods of optimal control of oscillations of systems with lumped and distributed parameters are shown in [5-7], where method of moments was used in the search of a control for deformable systems' oscillations.

In [8-10] results of searching of a wide class of skewsymmetric optimal controls for the translational motion of elastically deformable systems with a finite and infinite number degrees of freedom are presented, and examples of technical applications' characteristic are given. Papers [10-14] are devoted to the problems of optimal translational motion using the algorithm of the complete inverse problem of the variations calculus ("reversion calculus").

A generalization of the algorithm for the solutions of the complete inverse problem of the variations calculus (from a function to a functional) is the formulation of the reversion principle of optimality [11]. According to it, during minimal possible time (which is determined from the moment relations in the relative motion of the elastic system's period) restored (by a constructed motion) functional-criteria assumes a stationary value.

The experimental verification of the optimal translational parallel motion of an elastic system with one degree of freedom was performed for the first time [14] using FESTO laboratory equipment in Sevastopol State University (Sevastopol, Russia).
The purpose of the research is to use the previously developed technique of experimental research of the translational parallel motion of an elastic object to test the optimal translational rotary motion of a cantilever elastic rod with a distributed mass.

\section{Description of the experimental setup}

In Fig. 1 is a simplified block diagram of an experimental setup implementing optimal rotation for a rod about a fixed axis. The operating principle of the experimental setup's control system is based on implementation of the control law with the outputting of a command for the servo motor controller generating voltage of a certain frequency.

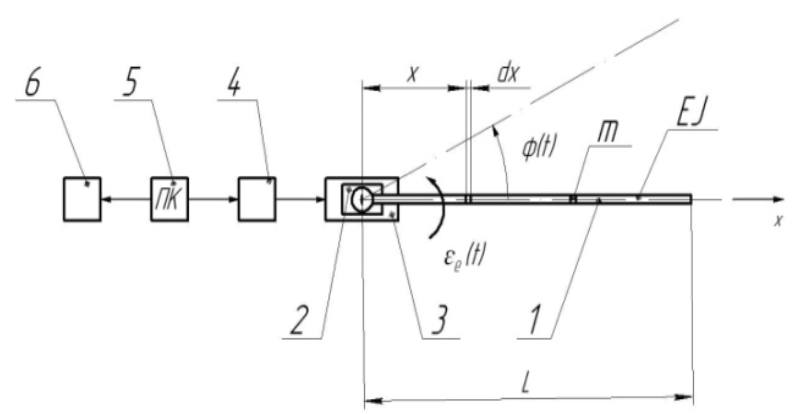

Fig. 1. Simplified scheme of the experimental setup: 1- cantilever rod with distributed mass; 2 - reduction gear; 3 - servomotor; 4 - servo controller; 5 - PC; 6- Arduino Mega 2560.

\footnotetext{
* Corresponding author: bohon.alex@mail.ru
} 


\section{Solution for the equations of the frame relative motion}

Similarly to the optimal translational motion described in [10], the acceleration of the translational rotary motion is taken the following form:

$$
\varepsilon_{e}(t)=a \cdot \sin (p t),
$$

where $a, p=$ const. The boundary conditions are given:

$$
\varphi_{e}(0)=0, \quad \dot{\varphi}_{e}(0)=0, \quad \varphi_{e}(T)=\varphi_{*}, \quad \dot{\varphi}_{e}(T)=0,
$$

where $T$ is a total time of a portable rotary motion, $\varphi_{*}$ is a maximum angle of rotation of the rod in the end of motion. After integrating (1), taking into account the boundary conditions (2), we obtain:

$$
\begin{gathered}
\varphi_{e}(t)=\frac{a}{p} \cdot\left(t-\frac{1}{p} \sin p t\right), \\
\varepsilon(t)=a \cdot \sin (p t), \quad \omega_{e}(t)=\frac{a}{p} \cdot(1-\cos p t)
\end{gathered}
$$

where $a=\frac{\varphi_{*} p^{2}}{2 \pi}$. When the rod had been rotated by an angle $\varphi_{*}$ during a time $T$, the translational rest would be achieved.

In the relative motion in the interval $T \geq t \geq 0$ flexural vibrations of the elastic rod could be observed. They are caused by the optimal translational rotary motion and could be described (without taking into account resistance to movement) by the following equation:

$$
E J \frac{\partial^{4} W(x, t)}{\partial x^{4}}+m \frac{\partial^{2} W(x, t)}{\partial t^{2}}=-f(x, t),
$$

where $E J$ is a rod's flexural stiffness ( $E-$ is Young modulus; $J$ - is an axial moment of inertia of rod's crosssection); $m-$ is a distributed mass $(\mathrm{kg} / \mathrm{m})$; $f(x, t)=\varepsilon_{e}(t) \cdot x \cdot m$, where $\varepsilon_{e}(t)=\frac{\varphi_{*} p^{2}}{2 \pi} \sin (p t)$ is a angular acceleration.

It is assumed that in this experiment the appearance of the elastic rod' first form (mode) of bending vibrations is most likely. According to the Fourier method [2, 3], taking into account the first mode of forced bending vibrations, following is accepted:

$$
W_{1}(x, t)=W_{1}(x) \cdot \sin (p t) .
$$

After substitution (5) in (4) and transformations, the fourth-order differential equation for the first mode of oscillation takes the following form:

$$
\frac{d^{4} W_{1}(x)}{d x^{4}}-k^{4} W_{1}(x)=-\frac{\varphi_{*} p^{2} m}{2 \pi \cdot E J}
$$

where $k^{4}=\frac{m p^{2}}{E J}$.

The solution of the inhomogeneous equation (6) is found using the functions of A.N. Krylov. The general solution of the inhomogeneous equation (6) could be written:

$$
\begin{aligned}
& W_{1}(x)=A \cdot s(x)+B \cdot T(x)+ \\
& C \cdot U(x)+D \cdot v(x)+\frac{\varphi_{*} p^{2} m}{2 \pi \cdot k^{4} E J},
\end{aligned}
$$

where

$$
\begin{aligned}
& s(x)=\frac{\cosh (k x)+\cos (k x)}{2} ; T(x)=\frac{\sinh (k x)+\sin (k x)}{2} ; \\
& U(x)=\frac{\cosh (k x)-\cos (k x)}{2} ; V(x)=\frac{\sinh (k x)-\sin (k x)}{2} .
\end{aligned}
$$

Taking into account the boundary conditions for the rod with a anchorage and the free end:

$$
\begin{aligned}
& W_{1}(0)=0, \quad W_{1}^{\prime}(0)=0, \\
& W^{\prime \prime}(L)=0, \quad W^{\prime \prime \prime}(L)=0 .
\end{aligned}
$$

After determining the constants A, B, C, D solution of equation (7) takes the following form:

$$
\begin{aligned}
& W=-\frac{1}{4} \frac{1}{k^{5}(1+\cosh (k L) \cdot \cos (k L)) \pi E I} \cdot \\
& \cdot\left(f p^{2} m(-2 k x \cdot \cosh (k L) \cdot \cos (k L)+\sin (k x) \cdot\right. \\
& \cdot \cosh (k L) \cdot \cos (k L)+\sinh (k x) \cdot \cosh (k L) \cdot \\
& \cdot \cos (k L)-\cos (k L) \cdot \sinh (k L) \cdot \cosh (k x)+ \\
& +\cos (k L) \cdot \sinh (k L) \cdot \cos (k x)+\cosh (k L) \cdot \\
& \cdot \sin (k L) \cdot \cosh (k x)-\cosh (k L) \cdot \sin (k L) \cdot \\
& \cdot \cos (k x)-\sinh (k L) \cdot \sin (k L) \cdot \sinh (k x)+ \\
& +\sinh (k L) \cdot \sin (k L) \cdot \sin (k x)-2 k x+\sinh (k x) \\
& W=-0.001262134811 \cdot \sinh (1.570796327 x)- \\
& -0.001262134811 \cdot \sin (1.570796327 x)+ \\
& +0.001376145229 \cdot \cosh (1.570796327 x)- \\
& -0.001376145229 \cdot \cos (1.570796327 x)+ \\
& +0.003965113454 x,
\end{aligned}
$$

where $k=\frac{\pi}{L}$.

For the numerical analysis with subsequent experimental verification, following initial data are accepted: specific weight of material is $\gamma=7800 \mathrm{~kg} / \mathrm{m}^{3}$; Young modulus is $E=2,1 \cdot 10^{11} \mathrm{~Pa}$; rod's diameter is $d=0,55 \cdot 10^{-2} \mathrm{~m}$; rod's length is $L=1 \mathrm{~m}$.

Calculated values are:

$$
m=\gamma \cdot \frac{\pi d^{2}}{4}=0,1853 \mathrm{~kg} / \mathrm{m}, J=\frac{\pi d^{4}}{64}=0,1853 M^{4} \text {. }
$$


According to [2], the frequency of the first tone of the oscillations is equal to $\omega_{1}=\frac{\lambda_{1}^{2}}{L^{2}} \sqrt{\frac{E J}{m}}=25.0 .8 \mathrm{~s}^{-1}$, where for the first mode $\lambda_{1}=1,875$ was accepted.

Period of oscillations' first tone is $T_{1}=\frac{2 \pi}{\omega_{1}} \approx 0.25 \mathrm{~s}$; further accepted: $p=\frac{\omega_{1}}{2}=12.54 s^{-1}$; so in this case the total motion time is $T=\frac{2 \pi}{p}=0.5 \mathrm{~s}$.

Graph of the function $W_{1}(x, t)=W_{1}(x) \cdot \sin (p t)$ when $T \geq t \geq 0$ is shown in fig.2.

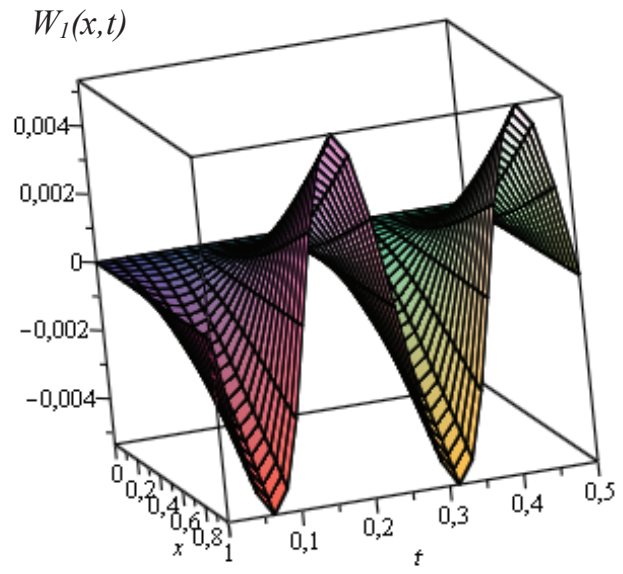

Fig. 2. Oscillations of the rod during relative motion

As it comes from the fig.2, at the moment $t=T$ comes relative rest, and, taking into account translational rest (fig.3), absolute rest will be achieved.
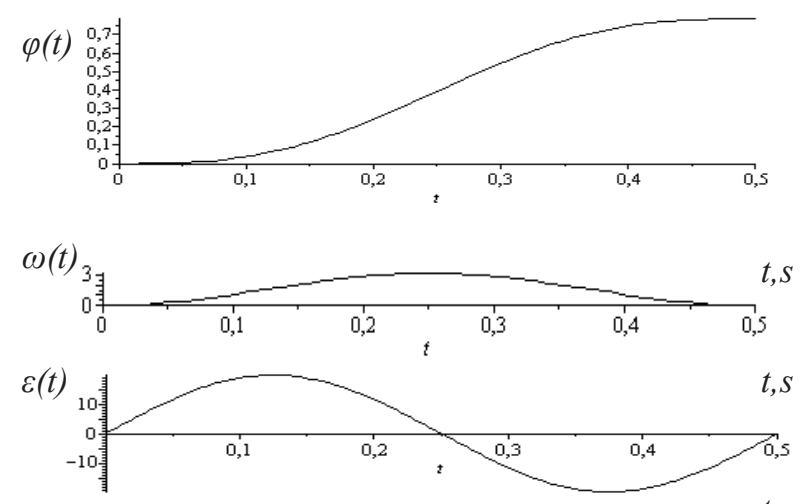

$t, s$

Fig. 3. Graphs: angle of rotation, angular velocity, angular acceleration

\section{The experimental procedure}

1. During the experimental research following equipment has been used: controller Simatic S7-300 CPU-313C; specialized controller SEC-AC-305 of the servomotor; rod with length $\mathrm{L}=1 \mathrm{~m}$ and diameter $\mathrm{d}=0,0055 \mathrm{~m}$; accelerometer MPU 6050, attached to the end of rod and connected to Arduino Mega 2560.

2. According to the program downloaded in the controller S7-300, the output voltage was calculated and applied to the input of the servo controller SEC-AC-305 and converted into the corresponding angular velocity of the output shaft of the servomotor.

3. Results of the experimental observations of relative oscillations were approximated by analytical function using MathCAD (Genfit function).

4. The maximum experimental relative displacements of the end of the rod are comparing with the theoretical ones, and the relative error is estimating.

\section{Conclusion}

The results of experimental studies confirm the practical possibility to carry out, with sufficient accuracy, skewsymmetric control laws of translational rotary motion of elastic objects with distributed parameters. Optimal motion controls for the objects with finite stiffness could be applied in a various field of technologies.

\section{References}

1. V.A. Svetlitsky, Collection of problems on the theory of oscillations (Vysshaya shkola, Moscow, 1973).

2. V.L. Biderman, Theory of mechanical oscillations (Vysshaya shkola, Moscow, 1980).

3. I.M. Babakov, Theory of oscillations (Nauka, Moscow, 1968).

4. Y.G. Panovko, Introduction to the theory of mechanical oscillations (Nauka, Moscow, 1980).

5. I.A. Karnovsky, Methods for optimal control of oscillations of deformable systems (Vishcha shkola, Kiev, 1982).

6. A.G. Butkovsky, Methods for managing systems with distributed parameters (Nauka, Moscow, 1975).

7. N.N. Krasovskii, Theory of motion control (Nauka, Moscow, 1968).

8. A.I. Bokhonsky, S.Y. Zolkiewski, Modeling and analysis of elastic systems in motion (Wydawnictwo Politechniki, Gliwice, 2011).

9. A.I. Bokhonsky, N.I. Varminskaya, Variational and reversion calculus in mechanics (Publishing house of SevNTU, Sevastopol, 2012).

10. A.I. Bokhonsky, N.I. Varminskaya, M.I. Mozolevsky, Optimum control of the translational motion of deformed objects: theory and technical applications (Publishing house of SevNTU, Sevastopol 2007).

11. A.I. Bokhonsky, Reversible Optimality Principle (Vuzovsky uchebnik: INFRA, Moscow, 2016).

12. A.I. Bokhonsky, Actual problems of variations calculus (Palmarium Academic Publishing, Saarbrücken, 2013).

13. A.I. Bokhonsky, A. Buchacz, M. Placzek, A.Wrobel, Modeling and investigation of discrete-continuous 
vibrating mechanic systems with damping (Wydawnictwo Politechniki, Gliwice, 2011).

14. A.I. Bokhonsky, M.M. Maistrishin, E.O. Balakanov, I.V. Gusakov, Experimental verification of optimal control of the translational motion of a deformable object, Optimization of production processes: collection of scientific papers, 12, pp.66-72, (2010). 